\title{
Applications of a generalized complementary energy principle for the equilibrium analysis of softening structures
}

\author{
Sheldon Plaxton, J.E. Taylor \\ University of Michigan, Ann Arbor, MI 481019, USA \\ Received 30 April 93 \\ Revised manuscript received 18 October 1993
}

\begin{abstract}
An interpretation for computational solution is given for a new global extremum principle that models a generalized form of elastic/softening structural behavior. The principle and its interpretation are expressed in a form that accommodates arbitrary heterogeneity and anisotropy in the structural material. Also the stress-strain properties that reflect evolution of local softening are represented in the model by a set of parameters defined over the field of the structure. Thus, the model may be used to predict the general behavior of solid structures having non-uniform stress/strain fields that evolve with change in external load. A discretized version of the principle used for computation is based on a consistent, mixed-form finite element interpretation of the principle as stated for the general softening continuum. Example computational solutions are provided covering the evolution of softening for a uniformly loaded homogeneous sheet with a hole, and simulations of a sheet with various configurations of softer or stiffer inclusions in an otherwise uniform structure.
\end{abstract}

\section{Introduction}

The purpose of this paper is to demonstrate the application of a recently developed extremum principle covering equilibrium problems for structures made of softening materials (as described for a one-dimensional stress state, a material for which 'change in stress per unit change in strain' diminishes as the magnitude of the load increases is referred to here as a softening material) [1]. The formulation makes use of a superposition of an arbitrary number of fields, here termed 'constituent fields,' to represent total stress, and this feature provides for a constructive approach to the simulation of stress-strain properties associated with any softening material. This representation of the total stress tensor is in contrast to a number of other approaches to the analysis of non-linear material response (e.g. [2-4]). With this construction, the non-linear problem is established in a form that in effect affords all of the advantages for analysis generally associated with having the continuum problem interpreted in terms of potentials.

For convenience, we first present a brief description of the principle stated for the non-linear continuum. An interpretation of the continuum model into discretized form is presented next. The discrete-form expression for the extremum problem statement is obtained using a self-consistent, mixed-form finite element construction. This leads to a convex constrained nonlinear programming problem. Computational solutions are obtained conveniently using standard software written for such problems. Numerical results are presented showing, for several example problems involving twodimensional softening continua, the evolution of softening and the corresponding stress and/or deformation fields under increasing load. These results are demonstrated for a rectangular sheet of bilinear softening material that has variously located sets of stiffer or less-stiff inclusions, and also for a rectangular sheet with a central hole, solved for each of two choices of softening materials. 


\section{The continuum model}

As indicated in the introduction, the present formulation of equilibrium analysis for softening materials is based on a construction where total stress is expressed as the sum of contributions from an arbitrary number of constituent fields. In the context of the variational problem, these stress constituent fields $\sigma_{i j}^{\gamma}$ and the field $u_{i}$ representing displacement appear as independent unknowns. The problem statement is given formally as (for simplicity, the formulation is expressed here for problems where specified boundary displacements have value zero):

$$
\min _{\alpha, u_{i}, \sigma_{l j}^{\gamma}} \frac{1}{2} \int_{\Omega}\left\{L_{i j k l} \boldsymbol{u}_{i, j} u_{k, l}+\sum_{\gamma=1}^{N} C_{i j k l}^{\gamma} \sigma_{i j}^{\gamma} \sigma_{k l}^{\gamma}\right\} \mathrm{d} V
$$

subject to:

$$
\begin{array}{ll}
\left(L_{i j k l} u_{k, l}+\sum_{\gamma-1}^{N} \sigma_{i j}^{\gamma}\right)_{. j}+\alpha X_{i}=0 & \text { in } \Omega, \\
\left(L_{i j k l} u_{k, l}+\sum_{\gamma=1}^{N} \sigma_{i j}^{\gamma}\right)_{j}-\alpha t_{i}=0 & \text { on } \Gamma_{i_{i}}, \\
f^{\gamma}\left(\sigma_{i j}^{\gamma}\right)-\overline{f^{\gamma} \leqslant 0} & \forall \gamma, \\
\underline{\alpha}-\alpha \leqslant 0 . &
\end{array}
$$

Scalar variable $\alpha$ represents a load factor, while $X$ and $t$ stand for a body force field and boundary tractions, respectively. Functions $f^{\gamma}\left(\sigma_{i j}^{\gamma}\right)$ by which the constituent fields $\sigma_{i j}^{\gamma}$ are bounded are limited only by the condition that the constraints where they appear in problem [P] must be convex. Loads $X$ and $t$, (constitutive) tensors $L$ and $C^{\gamma}$, and limits $\overrightarrow{f^{\gamma}}$ and $\underline{\alpha}$ are data. Note that the lead terms, i.e., the quantity within parentheses, in the first two constraints represents total stress. The quantity being minimized in $[\mathrm{P}]$ is the appropriate quadratic measure of fields $u_{k}$ and $\sigma_{i j}^{\gamma}$ associated with this 'total stress'. Within mild restrictions on the spaces for the fields named, the problem outlined in this statement is convex and therefore uniqueness of the solution is ensured. The net constitutive character is determined in this extremum problem statement via the combination of specified values for tensors $L$ and $C$, and the form of functions $f^{\gamma}$ and bounds $\bar{f}^{\gamma}$ in the constraints on the constituents $\sigma_{i j}^{\gamma}$. The model provides for the constructive simulation of material properties through the specification of these parameters, and the resulting stress-strain relation has a generalized polygonal form (a model for analysis of solids with piecewise linear material properties has been reported by Besseling [5]). We note that an entirely equivalent alternate formulation is available in the form obtained when the argument $\alpha$ of the fourth constraint in $[\mathrm{P}]$ is to be maximized within an upper bound constraint on the measure of energy appearing in the objective of problem $[\mathrm{P}]$ (the equilibrium constraints and constituent bounds are retained as is).

The maximizing fields and corresponding scalar value $\alpha$ associated with problem [P] comprise the solution to the cquilibrium problem represented there; details are given in [1]. The formulation is distinct from conventional expressions of variational models applicable to the analysis of such nonlinear systems (see e.g. [6]). It is unique by virtue of the feature mentioned above that makes it possible to synthesize the properties of a softening material, and also as a result of the representation of both stress and displacement within the single extremum problem statement. The multipliers associated with the set of constraints on the $\sigma_{i j}^{\gamma}$ fields are equivalent to what appear as internal variables in the usual model for systems with material degradation; in the present formulation they measure the differences betwcen total strain and 'linear strain' associated with each of the respective constituent stress fields. These details also are discussed in [1] on the basic formulation cited above.

It may be noted that in the present approach, numerical results are developed for the non-linear problem without the introduction of incremental modelling. Computational solutions are obtained for the several examples described in Section 4 by the application of a minimization program directly to the finite element interpretation of problem $[P]$. The evolution of behavior associated with the progressive 
softening of a structure, assuming given material and load configurations, is established simply through the results obtained for a set of solutions corresponding to an appropriate set of valucs for data item $\underline{\alpha}$. The constraint on $\alpha$ in [P] is necessarily active. For simplicity in what follows, the scalar $\alpha$ is eliminated and the remaining $\underline{\alpha}$ in fact represents specified load.

\section{Discrete formulation}

In this section, an approach is presented for numerical solution of the linear/softening boundaryvalue problem characterized via extremum statement $[\mathrm{P}]$ of Section 2. Introduction of discretized fields for both displacements and stress constituents leads to a matrix form statement for the discrete interpretation of the extremum principle. Here the matrix form is developed for a specific choice of discrete fields in a two-dimensional setting, and the methods used to obtain the numerical results presented in Section 4 are briefly discussed.

Let the domain $\Omega$ be represented by a collection of 3-node triangular elements with displacements interpolated linearly between element nodes, and let the $\sigma$ field(s) be approximated by piecewiseconstant shape functions, i.e., $\sigma_{i j}$ are constant within each element and are discontinuous at interclement boundaries. It is not necessary that the number of constituent fields in adjacent elements be the same. Thus, $u_{i} \approx \sum_{A=1}^{N_{\text {nod }}} N_{A} d_{i_{A}}$ and $\sigma_{i j}^{\gamma} \approx \sum_{B=1}^{N_{\text {sioc }}} \tilde{N}_{B} \sigma_{i j_{B}}^{\gamma}$ where $d_{i_{A}}$ is the displacement degree of freedom corresponding to Cartesian direction $i$ at node $A$ and $\sigma_{i j_{B}}^{\gamma}$ are the stress constituent components for the $\gamma$ th stress constituent on element $B . N_{\text {nod }}$ equals the total number of nodes and $N_{\text {sloc }}$ represents the total number of locations at which stress constituents are defined in the discretized model. Note that for this case of element-wise constant stress, only one term in the summation on $B$ is non-zero within a given element, while this is clearly not the case for a general discretization of the stress constituents fields.

Substitution of these discretized interpretations of stresses and displacements into the continuum problem statement $[\mathrm{P}]$ yields

$$
\min _{\{d\},\{\sigma\rangle} \int_{\Omega}\left\{\frac{1}{2} L_{i j k l}\left(\sum_{A=1}^{N_{\text {nod }}} N_{A, j} d_{i A}\right)\left(\sum_{I=1}^{N_{\text {nod }}} N_{I, l} d_{k_{l}}\right)+\frac{1}{2} \sum_{\gamma=1}^{N} C_{i j k l}^{\gamma}\left(\sum_{B=1}^{N_{\text {sloc }}} \tilde{N}_{B} \sigma_{i j_{B}}^{\gamma}\right)\left(\sum_{J=1}^{N_{\text {sloc }}} \tilde{N}_{J} \sigma_{k l_{J}}^{\gamma}\right)\right\} \mathrm{d} \Omega
$$

subject to:

$$
\begin{array}{ll}
\left(L_{i j k l}\left(\sum_{A=1}^{N_{\mathrm{nod}}} N_{A, l} d_{k_{A}}\right)+\sum_{\gamma=1}^{N}\left(\sum_{B=1}^{N_{\text {sloc }}} \tilde{N}_{B} \sigma_{i j_{B}}^{\gamma}\right)\right)_{, j}+\underline{\alpha} X_{i}=0 & \text { in } \Omega, \\
\left(L_{i j k l}\left(\sum_{A=1}^{N_{\text {nod }}} N_{A, l} d_{k_{A}}\right)+\sum_{\gamma=1}^{N}\left(\sum_{B=1}^{N_{\text {sloc }}} \tilde{N}_{B} \sigma_{i j_{B}}^{\gamma}\right)\right)_{j}-\bar{\alpha} t_{i}=0 & \text { on } \Gamma_{t_{i}}, \\
f^{\gamma}\left(\sum_{B=1}^{N_{\text {sloc }}} \tilde{N}_{B} \sigma_{i j_{B}}^{\gamma}\right)-\tilde{f}^{\gamma} \leqslant 0 & \text { in } \Omega,
\end{array}
$$

where $\{d\}$ and $\{\sigma\}$ are the vectors of unknowns in the discrete representation. Multiplication of the equilibrium equation by a virtual displacement field given as $v_{i}=\sum_{D=1}^{N_{\text {nod }}} N_{D} c_{i_{D}}$, where the nodal virtual displacements $c_{i_{D}}$ are zero at locations where displacements are specified, and integration over the domain $\Omega$ leads (after integration by parts and substitution of the boundary condition equations) to

$$
\begin{aligned}
c_{i_{D}} & \left\{\int_{\Omega}-N_{D, j} L_{i j k l}\left(\sum_{A=1}^{N_{\text {nod }}} N_{A, l} d_{k_{A}}\right) \mathrm{d} \Omega-\int_{\Omega} N_{D, j} \sum_{\gamma=1}^{N}\left(\sum_{B=1}^{N_{\text {sloc }}} \tilde{N}_{B} \sigma_{i j_{B}}^{\gamma}\right) \mathrm{d} \Omega\right. \\
& \left.+\int_{\Omega} N_{D} \underline{\alpha} X_{i} \mathrm{~d} \Omega+\int_{\Gamma_{t_{i}}} N_{n} \underline{\alpha} t_{i} \mathrm{~d} \Gamma\right\}=0 .
\end{aligned}
$$

Since this statement is to hold for arbitrary values of the $c_{i_{D}}$, a linear equation in $\{d\}$ and $\{\sigma\}$ results for each unspecified nodal displacement $d_{i_{A}}$. With the application of suitable numerical integration, the 
above equation then defines matrices $[K]$ and $[S \mid$ and the vector $\{p\}$ of equivalent nodal forces consistent with the matrix representation of equilibrium stated as follows:

$$
[K]\{d\}+[S]\{\sigma\}-\{p\}=\{0\} .
$$

Due to the choice of piecewise constant shape functions $\tilde{N}$ for the stress constituents, it is observed from the discrete form statement [D] that the inequality constraint $f^{\gamma}\left(\sigma_{i j_{\beta}}^{\gamma}\right)-\overline{f^{\gamma}} \leqslant 0$ is enforced element-wise, rather than pointwise, in the discretized domain. Thus, the simpler notation $f_{i}\left(\sigma_{i}\right)-\bar{f}_{i} \leqslant 0$ is adopted here to describe the stress bound constraints in the discrete form, where the variable $i$ refers to an individual local stress tensor $\sigma_{k i}^{\gamma}$ at the $i$ th element. As with the inequality constraints, the contribution per stress constituent to the coefficient matrix of the objective function may be reduced to an integral over each element domain of the corresponding compliance tensor $C$, the resulting block diagonal matrix being denoted by $[\tilde{C}]$. The displacement term in the objective results from the inner product of the vector of nodal displacements and the first term in F.q. (2) with a constant multiplier, and thus the complete matrix form of the objective is

$$
\frac{1}{2}\{d\}^{\mathrm{T}}[K]\{d\}+\frac{1}{2}\{\sigma\}^{\mathrm{T}}[\tilde{C}]\{\sigma\} .
$$

The complete discrete problem statement $[D]$ may now be expressed in the following form:

$$
\min _{\{d\},\{r\}}\left\{\frac{1}{2}\{d\}^{\mathrm{T}}[K]\{d\}+\frac{1}{2}\{\sigma\}^{\mathrm{T}}[\tilde{C}]\{\sigma\}\right\}
$$

subject to:

$$
\begin{aligned}
& {[K]\{d\}+[S]\{\sigma\}-\{p\}=\{0\},} \\
& f_{i}\left(\sigma_{i}\right)-\bar{f}_{i} \leqslant 0 .
\end{aligned}
$$

With the previously mentioned restriction of positive definiteness of constituent tensors, it may be noted that $[K]$ is a sparse symmetric positive definite matrix, $[\tilde{C}]$ is positive definite and block diagonal, and $[S]$ is a sparse rectangular matrix which relates stress constituent degrecs of freedom to corresponding nodal forces. The same form of problem statement is obtained for more general choices of shape functions $N$ and $\tilde{N}$, provided stress constituent bounds are enforced at 'stress node' locations. Also, relaxation of the assumption of zero values for enforced displacements would result in an additional linear term in the objective of the discrete problem statement, accompanied by the usual update of the force vector $\{p\}$.

The discrete form of the problem as expressed in $\left[\mathrm{D}^{\prime}\right]$ is amenable to solution by any nonlinear programming approach. For the particularly simple case of independent bounds on the separate components of constituent stresses, the formulation amounts to a straightforward quadratic programming problem. Another possibility which results in a quadratic programming problem is to approximate the nonlinear form of bound constraints by a set of linear bounds on the constituents, as has been considered in some earlier works (e.g. [7]). More generally, statement [D'] represents a fairly simple form of nonlinearly constrained minimization problem. The results of Section 4 represent the implementation of a complementary energy density form of stress constituent bound. Since computational requirements for nonlinear programming are generally proportional to both the total number of constraint equations and the number of unknowns, it is advantageous to consider reducing either of these quantities if possible. In the form given by $\left[\mathrm{D}^{\prime}\right]$, the stress-limiting constraint functions $f_{i}\left(\sigma_{i}\right)$ are independent of the displacement variables $d$ (although there may be reason to consider functions in the more general form $f(\sigma, d)$ ). This fact, coupled with the linearity of the equilibrium equations makes it possible to eliminate displacement variables from the actual (computational) minimization problem. Specifically, one may substitute for displacement according to $\{d\}=[K]^{-1}(\{p\}-[S]\{\sigma\})$, whereby the formulation $\left[D^{\prime}\right]$ is reduced to the form

$$
\min _{\{;\}}\left\{\frac{1}{2}\{\sigma\}^{\mathrm{T}}\left[[S]^{-\mathrm{T}}\left[\left.K\right|^{-\mathrm{T}}[S]+[\tilde{C}]\right]\{\sigma\}-\{p\}^{\mathrm{T}}[K]^{-\mathrm{T}}[S]\{\sigma\}\right\}\right. \text {. }
$$

subject to:

$$
f_{i}\left(\sigma_{i}\right)-\bar{f}_{i} \leqslant 0
$$


The corresponding displacement solution is then obtained by backsubstitution as a postprocessing step. It should be clear from [R] that a significant reduction in solution effort is possible if a portion of the domain is known to be linearly elastic, or to remain in the linearly elastic region throughout the load range of interest.

The solutions for the numerical examples discussed in this paper were obtained using form [R] and the Fortran code NLPQL, an established subroutine developed by Schittkowski [8] for the treatment of the nonlinear programming problem via a sequential quadratic programming approach. NL.PQL provides a solution vector which satisfies, to a user-specified tolerance, the necessary conditions for a local minimum. External to NLPQL, an automatic scaling algorithm was implemented in order to increase the rate of convergence and improve the condition of the matrices which are defined in the problem statement. This scaling, although routine in form, is a significant factor in the performance of the present numerical implementation of the extremum problem statement. As discussed previously, the convexity of the problem ensures that the local minimum is in fact a global minimum, and thus is the desired solution. Other solution approaches, perhaps taking advantage of the sparsity present in $\left[D^{\prime}\right]$ but not in $[R]$, are not considered here.

\section{Numerical examples}

A number of simulations are presented in this section to demonstrate the application of statement [R], or equivalently [D'], for equilibrium analysis of structures composed of softening materials. In each of these examples the softening materials are simulated using just two isotropic constituents. The specific properties of the constructed material depend of course on the individual constituent properties as well as the form of the stress constituent limit. Poisson's ratio is taken to be 0.3 in each of the constituents of these examples, and as mentioned in Section 3, the complementary energy density form of stress constituent bound is used. The properties of the simulated material are then adequately characterized by the value of the combined moduli, and the modulus in the softened state (when the stress constituent bound is active). For the first several examples, an end loaded rectangular sheet with one of three given configurations of inclusions is analyzed using the finite element discretization shown in Fig. 1. The linear/softening material of the sheet has a $90 \%$ reduction in modulus upon softening, while the inclusions are modelled as linear material, and are either stiff of soft relative to the initial modulus of the sheet. Specifically, for the initial modulus of the sheet identified in terms of the elasticity tensor $L$ and stress constituent compliance tensor $C$ as $L_{i j k l}+C_{i j k l}^{-1}$, the inclusions are taken to have constant constituent tensor $\mu\left(L_{i j k l}+C_{i j k l}^{-1}\right)$ where $\mu>1$ for a stiff inclusion and $0<\mu<1$ for a soft inclusion. The second group of results compares the response of an edge-loaded rectangular membrane with a central circular hole for two linear/softening materials with different softening behavior. The finite element discretization and boundary conditions for these examples are indicated in Fig. 2.

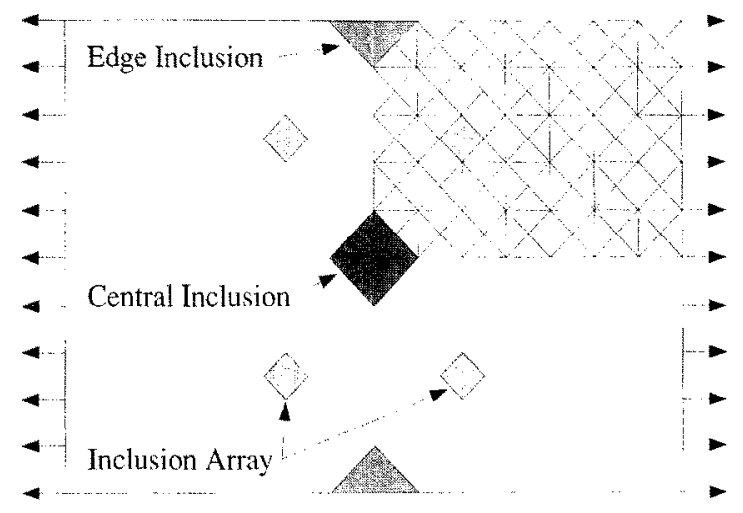

Fig. 1. Rectangular sheet layout with inclusion locations. 


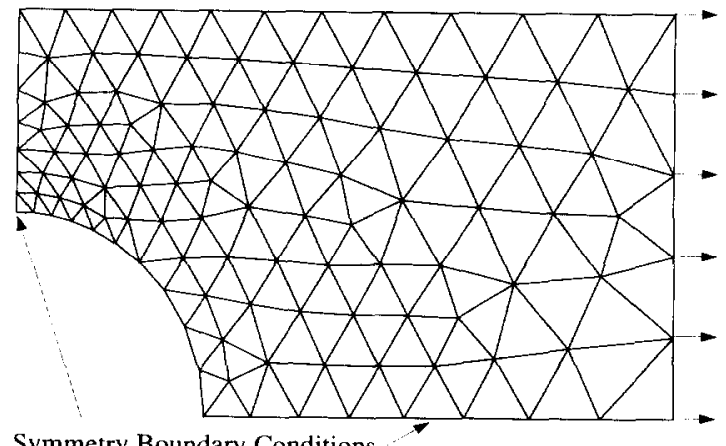

Symmetry Boundary Conditions

Fig. 2. Edge-loaded uniform membrane with central hole.

\subsection{Central inclusion in a rectangular sheet}

Two cases are considered for the elastic central inclusion: a soft central inclusion with modulus equal to one-half the initial modulus of the rest of the sheet, and a stiff central inclusion with modulus equal to twice the initial value of the softening material. In each case a uniform edge load per unit length is applied as indicated in Fig. 1.

\subsubsection{Soft central inclusion}

Computational results are presented in two types of plots. For the examples involving inclusions, plots are displayed for the full four-quadrant model in order to provide better visualization of the results. The first type of a plot shows the evolution of the softened region by simply identifying which elements have softened at a number of selected load values. Fig. 3(a), for example, depicts the

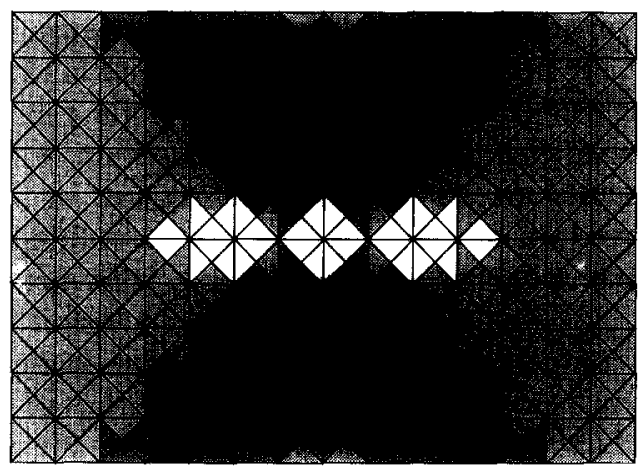

(a)
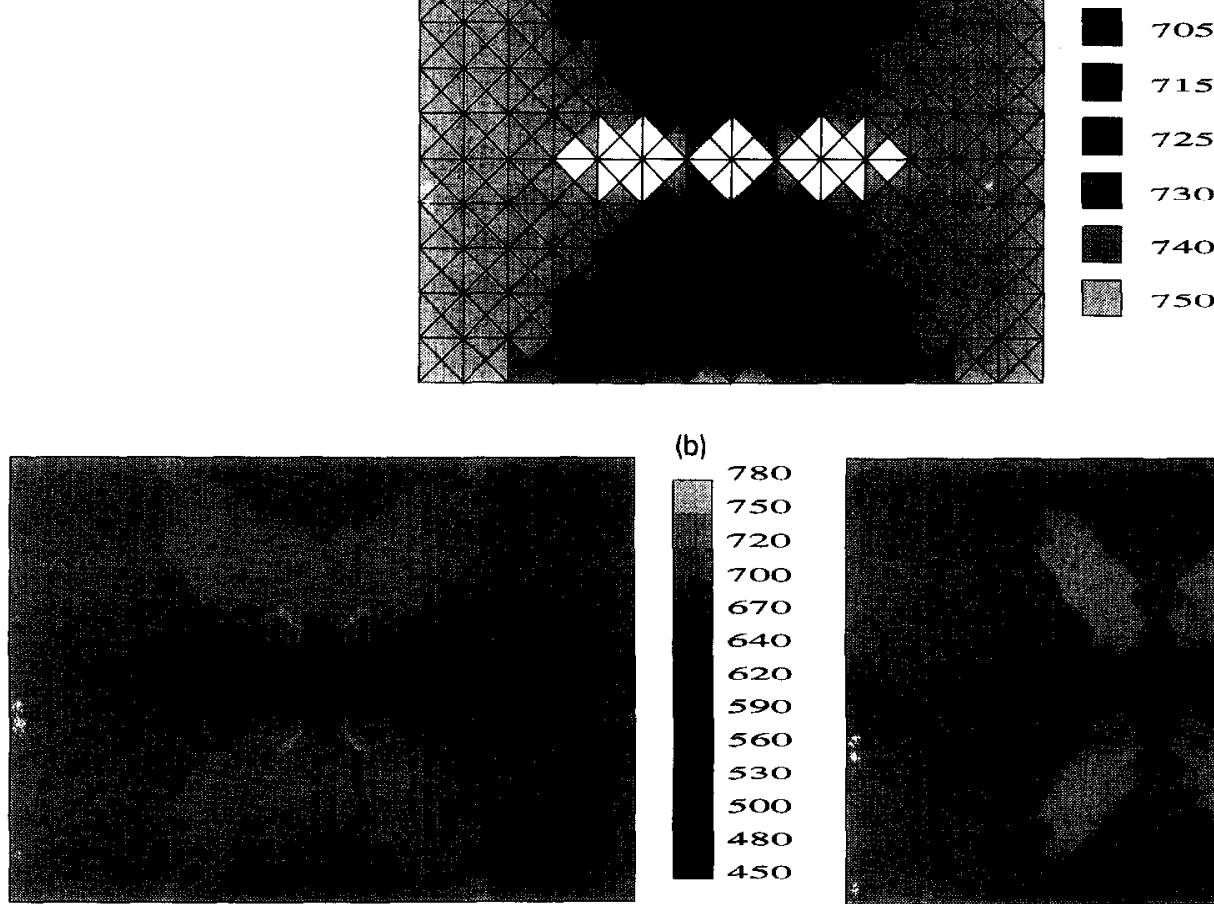

(b)
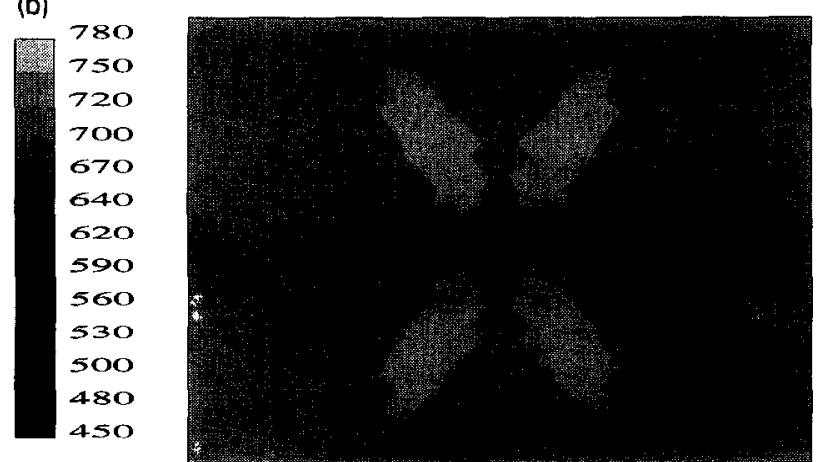

(c) 780 750 720 700 670 640 620 590 560 530 500 480

Fig. 3. Results for soft central inclusion. (a) Evolution of softening. (b) Effective stress contour for load $=715$. (c) Effective stress contour for load $=740$ 
evolution of softening for the case of a soft central inclusion. The shading in the figure is interpreted as follows: considering the level of grey associated with a particular load, all elements of that shade and darker will have softened at the current load level. Fig. 3(a) indicates the onset of softening in elements near the inclusion for a load value of 705 , and the progressive spread of softening for a number of load values up to the final displayed load of 750 . At the highest load shown, it is clear that nearly all of the material in the sheet has softened, and the location of the remaining unsoftened material indicates a 'shielding' effect, i.e., an effect which has the lower stiffness of the soft inclusion result in a lower stress level in the adjacent material along the direction of primary axial load.

Contours of effective stress (or strain, as indicated) are given in the second type of plot. The effective stress, defined in terms of principal stresses $\sigma_{\mathrm{I}}$ and $\sigma_{\mathrm{II}}$ by $\sigma_{\text {eff }}=\left(\frac{1}{2}\left\{\left(\sigma_{\mathrm{I}}-\sigma_{\mathrm{II}}\right)^{2}+\sigma_{\mathrm{I}}^{2}+\sigma_{\mathrm{II}}^{2}\right\}\right)^{1 / 2}$ (and similarly for strain), is interpolated linearly between element centroids to provide reasonable contours of the results. Effective stress contours for the soft central inclusion are given for two values of load in Figs. 3(b) and (c). The results emphasize the progressive development of regions of increased stress in a direction oblique to the load axis.

\subsubsection{Stiff central inclusion}

The evolution of the softened region is displayed for this case in Fig. 4(a). The figure indicates that initial softening occurs adjacent to the inclusion and along the axis of applied load, followed by progressive softening at the sheet boundary above and below the inclusion. The shielding effect in this case is reversed in that the stiffer inclusion results in a region of relatively lower stress in adjacent material oblique to the direction of primary tensile load, and an area of unsoftened material remains directly above and below the inclusion. Concentration of stress in the stiff inclusion is noticeable in the stress contours of Fig. 4(b) and (c), as is the shielding effect. Portrayals of the results for stiff and soft inclusions are somewhat complementary in terms of their topological features.

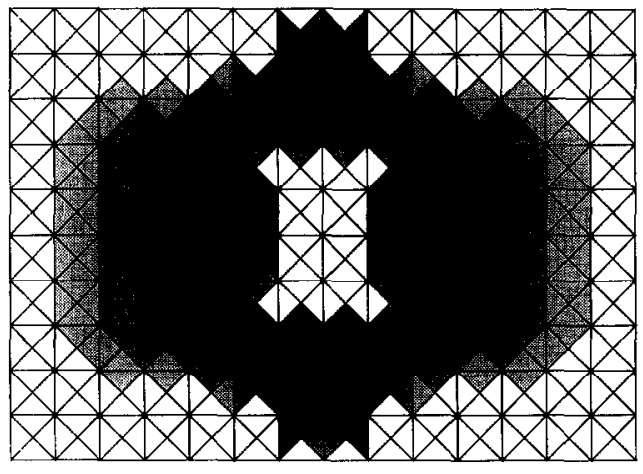

(a)
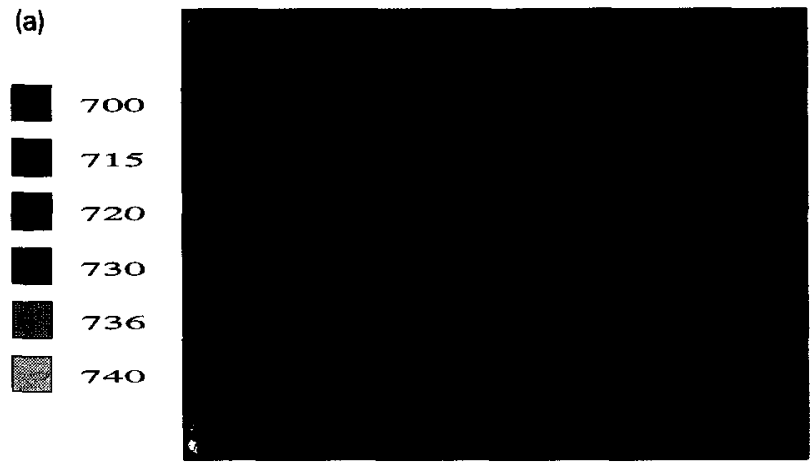

(b)
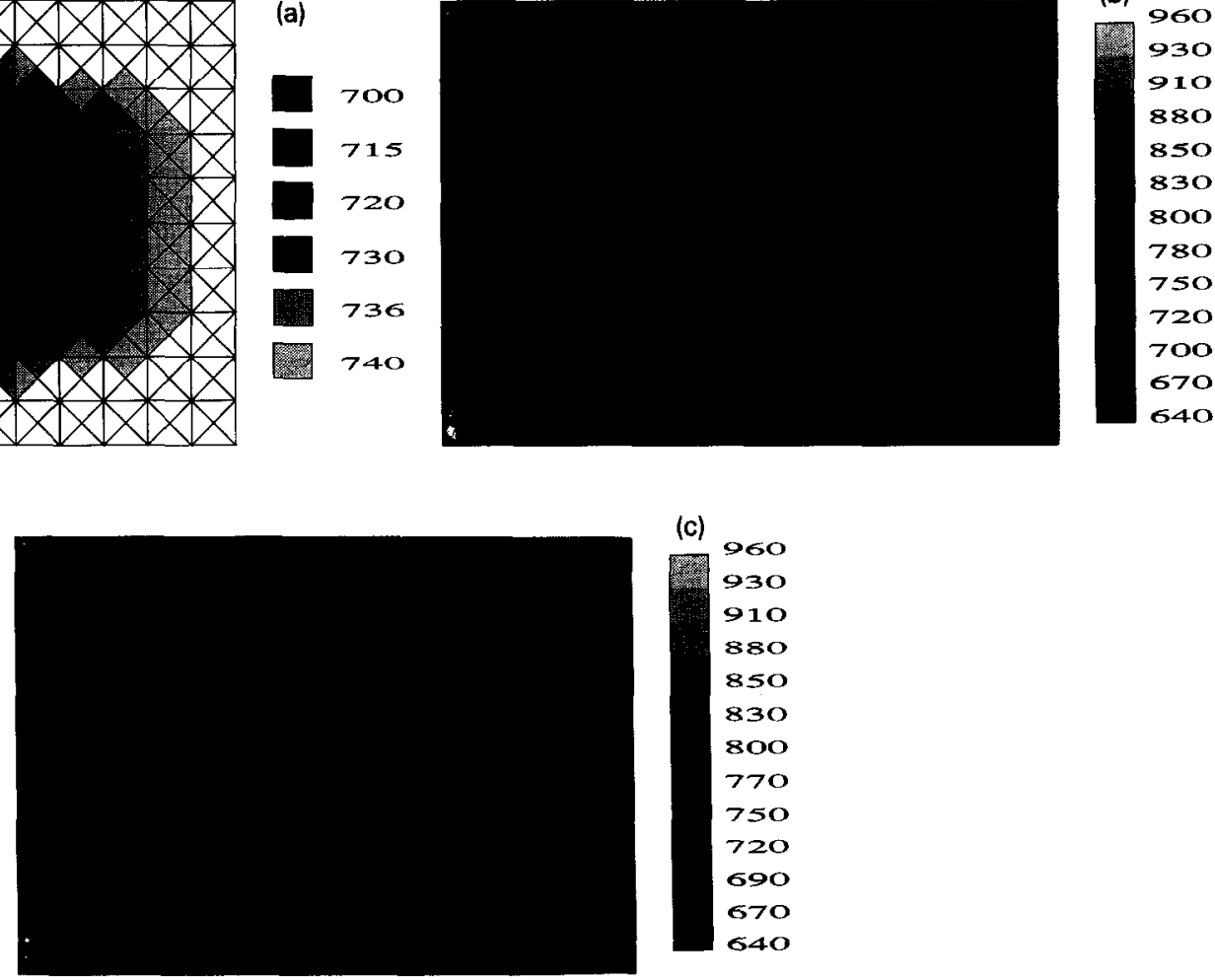

Fig. 4. Results for stiff central inclusion. (a) Evolution of softening. (b) Effective stress contour for load $=700$. (c) Effective stress contour for load $=736$. 


\subsection{Edge inclusions in a rectangular sheet}

The relative material properties for the two cases considered here are identical to those for the central inclusion examples discussed previously. In this case, however, the inclusions are located at the two edges of the sheet, as indicated in Fig. 1.

\subsubsection{Soft edge inclusions}

The progression of the softened region for soft edge inclusions is shown in Fig. 5(a). The evolution in this case is oblique to the axis of applied load, and shows similarity to that for the soft central inclusion discussed in Section 4.1.1. The shielding effect is again apparent in both the softening evolution and the stress contours (Figs. 5(b) and (c)). The contours indicate a progression towards a region of uniformly increased effective stress between the two edge inclusions. It is reasonable to expect, however, that the eventual trend would be similar to that for stiff edge inclusions, since after sufficient softening, the clastic inclusions are indeed stiffer than most of the rest of the sheet.

\subsubsection{Stiff edge inclusions}

The evolution of the softened region, shown in Fig. 6(a), and the stress contours of Fig. 6(b) and (c) again indicate the complementary nature of results for the stiff inclusions compared to those of the soft inclusions. Note that the shading scheme is reversed in the two contour plots relative to previous plots. A region of reduced effective stress is clearly visible in both the final plot of the softened region, and in the stress contour for the higher load.

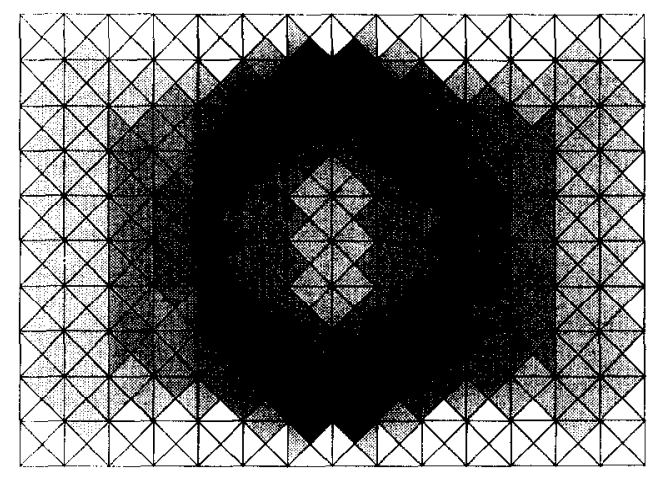

(a)
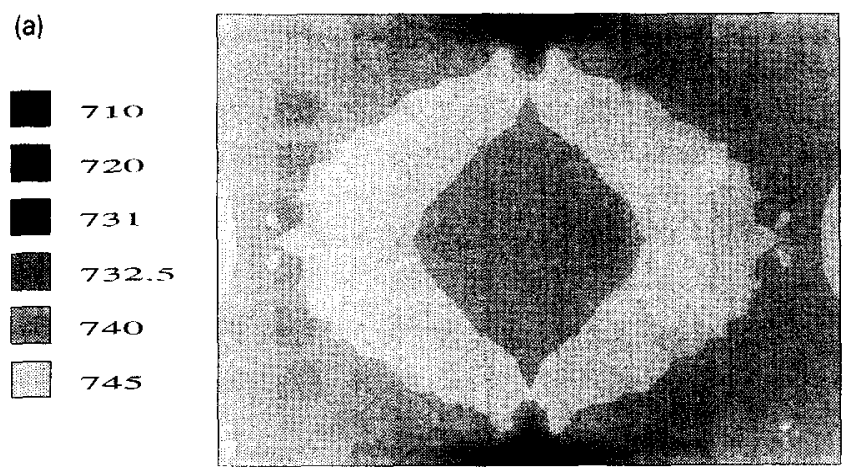

(b) 750

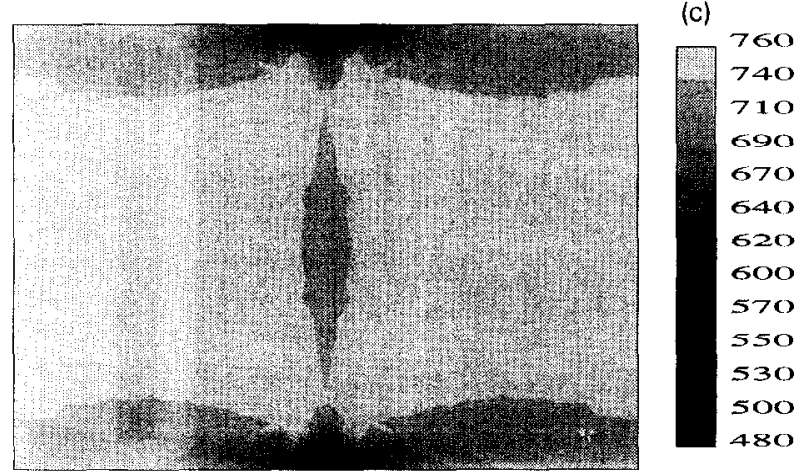

Fig. 5. Results for soft edge inclusions. (a) Evolution of softening. (b) Effective stress contour for load $=720$. (c) Effective stress contour for load $=737.5$ 

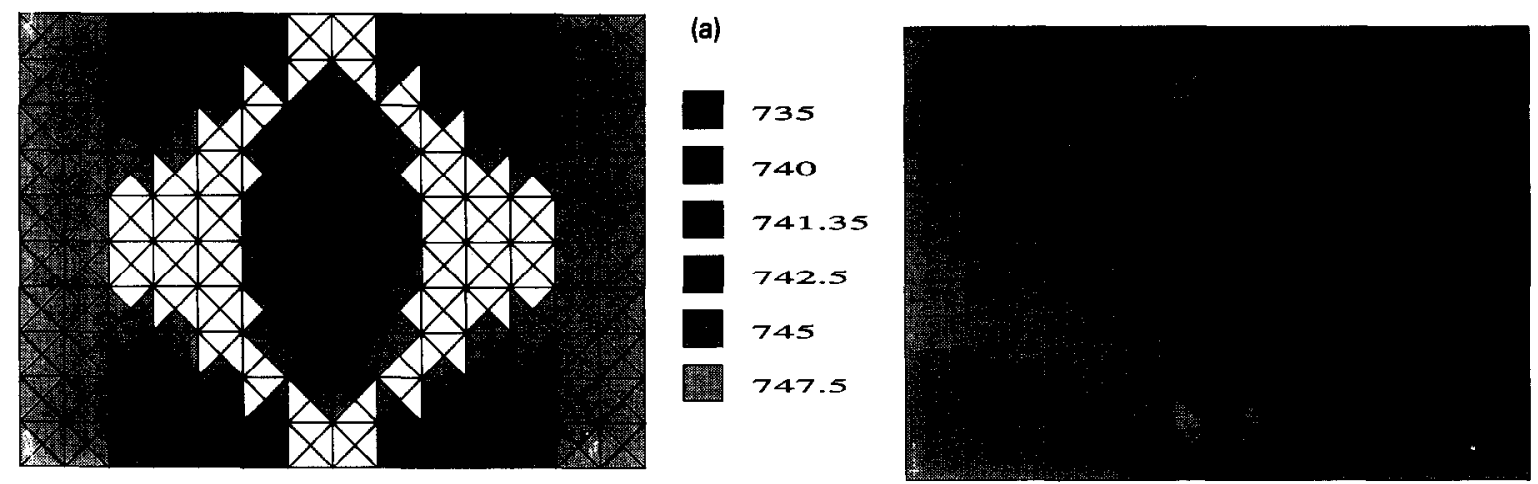

(b)

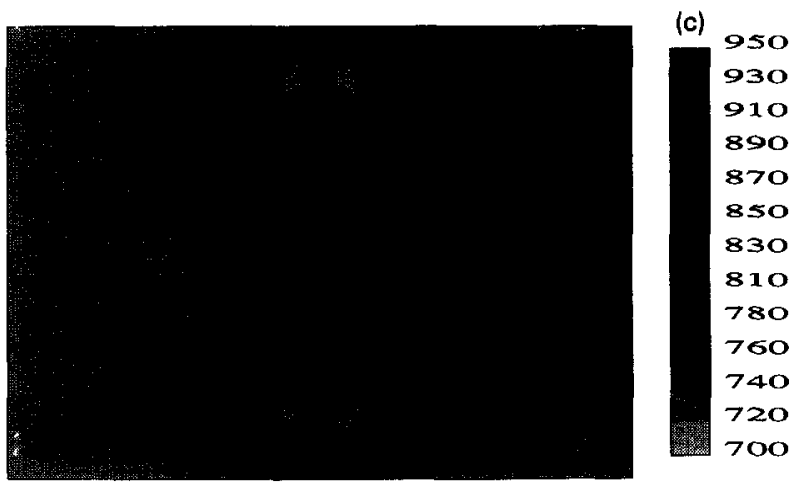

Fig. 6. Results for stiff edge inclusions. (a) Evolution of softening. (b) Effective stress contour for load =735. (c) Effective stress contour for load $=747.5$.

\subsection{An array of soft inclusions in a rectangular sheet}

In this simulation, four elastic inclusions are located in an array as shown in Fig. 1, and are taken to have a stiffness equal to one-tenth the initial stiffness of the surrounding domain. The evolution of softening, given in Fig. 7(a), is quite similar to that for the previously considered examples with soft inclusions, with the exception of the increased interaction of softened regions present in this example. The effective strain contours displayed in Fig. 7(b) and (c) clearly show the effect of interactions of the strain field disturbances due to each inclusion on the resulting strain distribution.

\subsection{Sheet with central hole}

In this example, a simulation is performed for the membrane shown in Fig. 2 with hole diameter equal to one-half the membrane width. Two sets of results are obtained, each set corresponding to a distinct choice of material in the sheet. The first material is characterized by a bilinear response under uniaxial load, such that the modulus after the onset of softening is $50 \%$ of the initial modulus. The second material behaves identically as the first up to the point of softening, after which its modulus is $1 \%$ of the initial modulus. In the following discussion, the respective materials are identified as $50 \%$ dropoff and $99 \%$ dropoff.

Figs. 8(a) and 9(a) show the progressive softening for the 50\% dropoff and $99 \%$ dropoff materials, respectively. In each case, the range of loads displayed is chosen such that a significant portion of the domain has softened. Of interest in the figures is the difference in the softening trend between the two materials, especially in the earlier stages of the load evolution. Specifically, one may observe that the boundary of the softened region for the more drastically softening material progresses, at least initially, in a comparably narrow region along the vertical centerline of the sheet. In comparison, the softened 


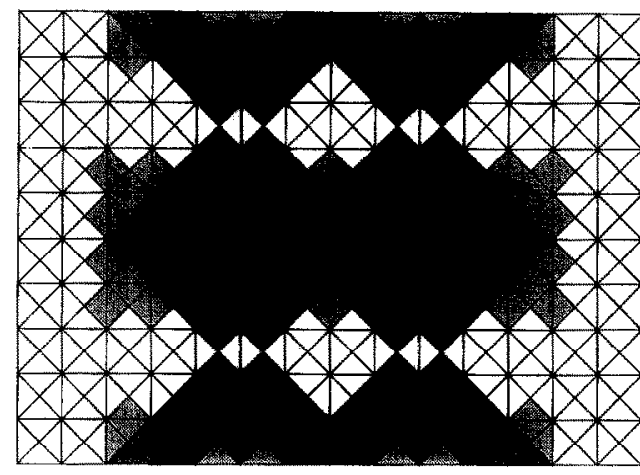

(a)

650

675

700

710

720

740

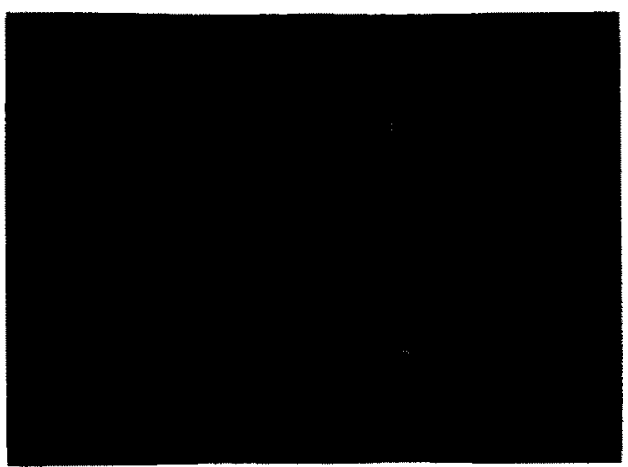

(b)
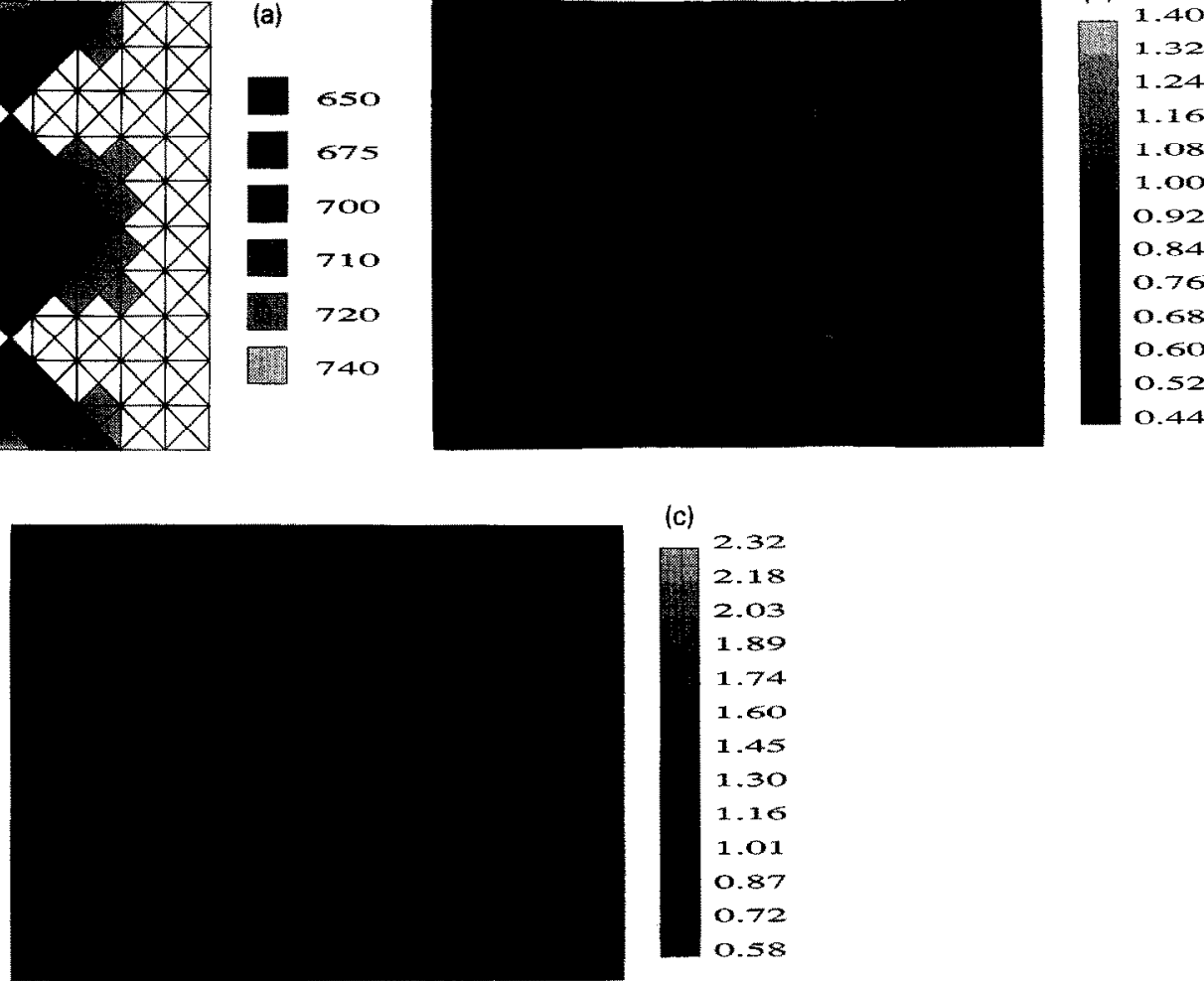

(c)

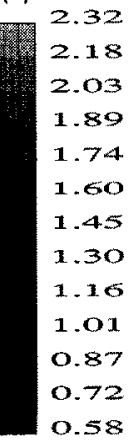

Fig. 7. Results for soft inclusion array. (a) Evolution of softening. (b) Effective strain $\left(\times 10^{+3}\right)$ contour for load $=650$. (c) Effective strain $\left(\times 10^{+3}\right)$ contour for load $=730$.

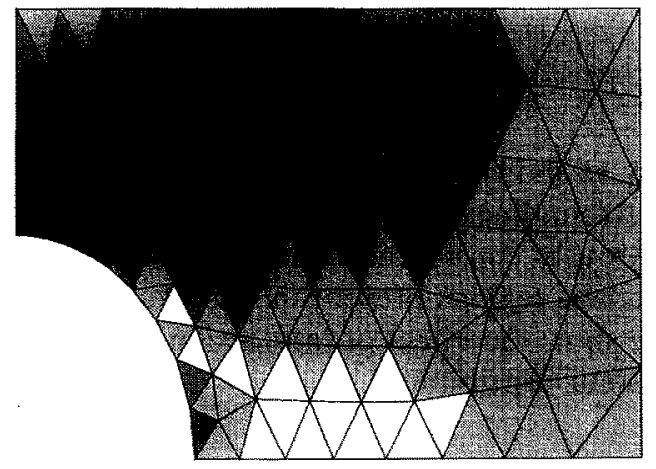

(a)
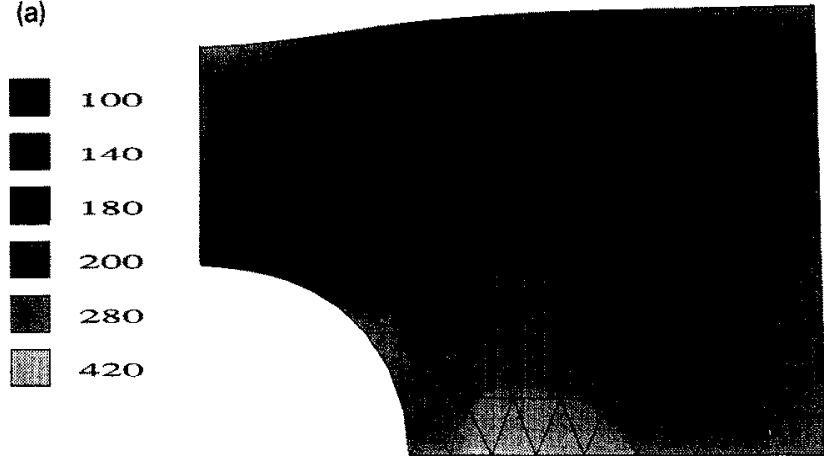

(b)

940
860
790
720
650
570
500
430
360
290
210
140
68

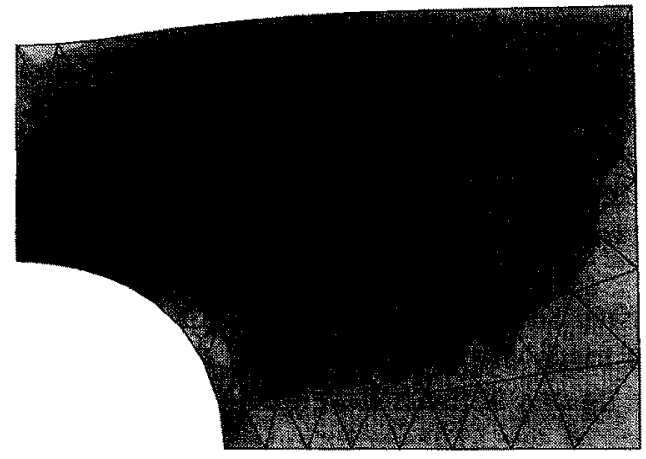

(c)

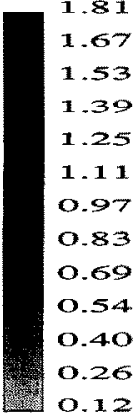

Fig. 8. Results for $50 \%$ dropoff material. (a) Evolution of softening. (b) Effective stress contour for load $=240$, deformed shape $\times 250$. (c) Effective strain $\left(\times 10^{+3}\right)$ contour for load $=240$, deformed shape, $\times 250$. 


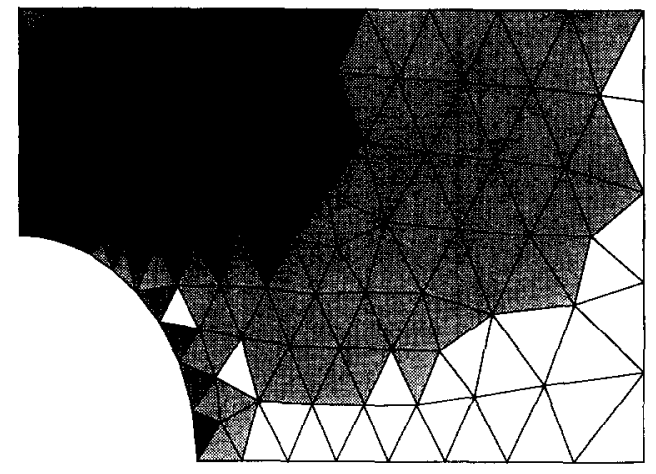

(a)

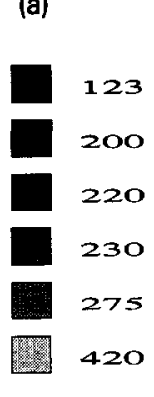

(b)
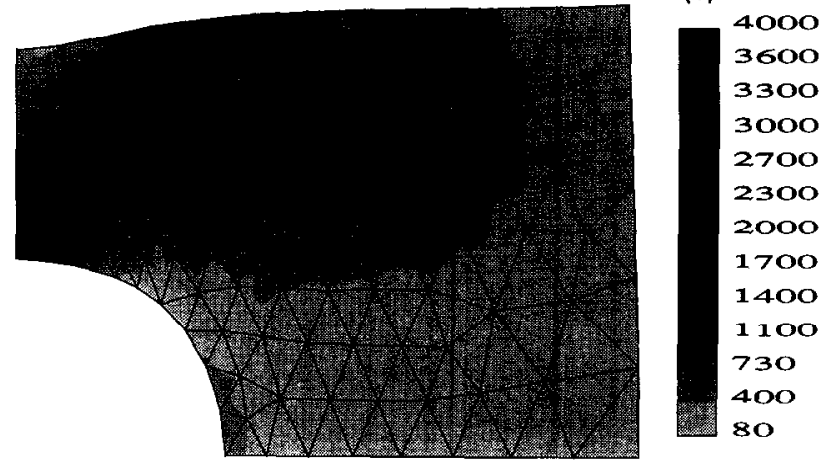

(c)

8.30
7.60
6.98
6.28
5.59
4.89
4.19
3.49
2.79
2.10
1.40
0.72
0.042

Fig. 9. Results for $99 \%$ dropoff material. (a) Evolution of softening. (b) Effective stress contour for load $=230$, deformed shape $\times 150$. (c) Effective strain $\left(\times 10^{+3}\right)$ contour for load $=230$, deformed shape, $\times 150$.

region for the $50 \%$ dropoff material is more diffuse, although the differences are less apparent at the higher load levels. Fig. 8(b) and (c) provide examples of effective stress and effective strain contours, respectively, for the $50 \%$ dropoff material. The stress contour shows the predictable concentrations about the hole, while the strain contour demonstrates both the localization of strain at the top of the hole and the presence of a lateral shielding effect. The (exaggerated) deformed shape of the membrane is also shown in both of the contour plots. Figs. 9(b) and (c) present comparable results for the $99 \%$ dropoff material. Finally, Fig. 10 displays a comparison of the overall response of the membrane for the two material types.

\section{Discussion}

A numerical implementation of an extremum principle for the equilibrium analysis of structures composed of linear/softening materials has been presented and applied to several computational examples. At this point, it is instructive to consider possible refinements in the present approach, as well as to discuss a number of directly available extensions and applications not covered by the examples. It is clear that significant refinement of the computational approach is possible. For example, the choice of discretization structure used in the examples is admittedly coarse. Further, certain improvements in computational efficiency would be achieved by using a computational approach tailored to the specific functional forms involved in this problem.

Note that the general statement of the extremum principle for the continuum, $[\mathrm{P}]$, involves a set of very general parameters $L_{i j k l}, C_{i j k l}^{\gamma}, \overline{f^{\gamma}}$ along with the choice of the number $N$ of local constituents. These parameters allow for 'fitting', either in a least squares sense or according to some other criterion, of properties for softening materials over any range of interest. The computational examples presented in Section 4 exemplify application of the model based on the use of two isotropic constituents for 


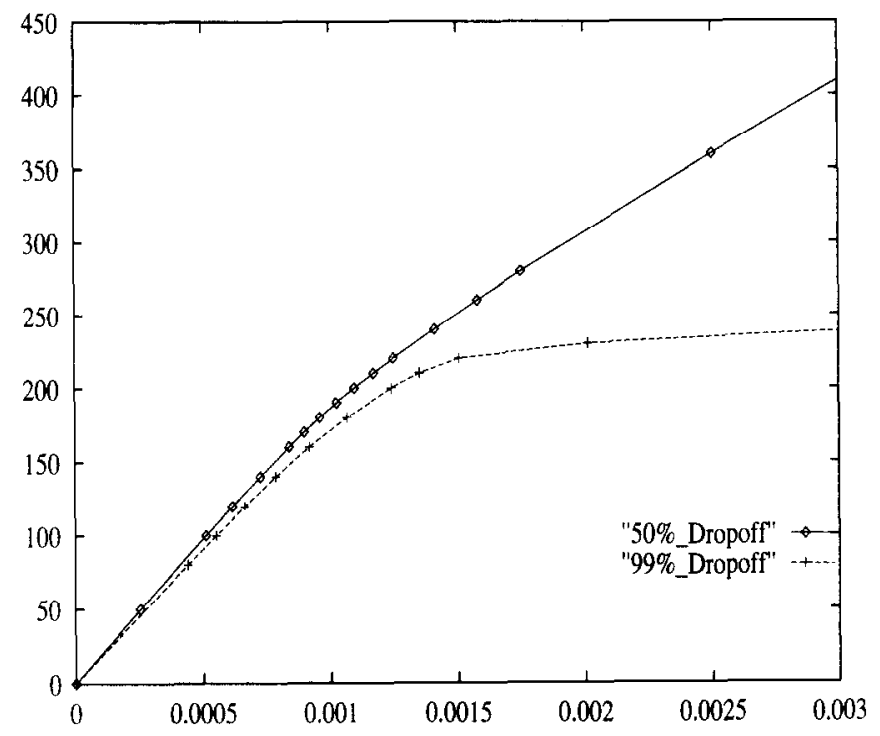

Fig. 10. Load versus end deflection comparison.

approximating material response. However, arbitrary softening material properties are accommodated within the model, the only restriction on the problem parameters being those necessary to maintain convexity in the global sense. Specifically, the restrictions are: (1) positive definiteness of the elastic constituent tensor $L,(2)$ convexity of the stress constituent potentials defined previously assuming linear elasticity, but further generalizable to $U^{*}\left(\sigma_{i j}^{\gamma}\right)$ for non-linear constituent response, and (3) convexity of the stress constituent constraints $f^{\gamma}\left(\sigma_{i j}^{\gamma}\right)$. The load paths considered up to this point have been strictly proportional. For the non-linear elasticity discussed in this paper, it is straightforward to accomodate any form of load path comprised of a sequence of segments, where the loading is proportional along each segment. This follows simply from the property that the formulation [P], valid for proportional loading, is convex. As an example of its application, the model for analysis of systems including softening material has been extended to the treatment of optimal design of truss structures $[9,10]$.

In summary, the constructive approach to representing general constitutive relations in the model provides for a substantial degree of flexibility in the method. The basic form of the extremum problem statement facilitates straightforward implementation of means to obtain numerical solutions.

\section{Acknowledgment}

The authors wish to thank Mr. Klaus Schittkowski for his helpful discussions regarding the use of the NLPQL routine. Also, the support of the first author by the National Science Foundation Graduate Fellowship Program is greatly appreciated.

\section{References}

[1] J.E. Taylor, A global extremum principle for the analysis of solids composed of softening material, Internat. J. Solids Struct. 30 (1993) 2057-2069.

[2] J.C. Simo, J.G. Kennedy and R.L. Taylor, Complementary mixed finite element formulations for elastoplasticity, Comput. Methods Appl. Mech. Engrg. 74 (1989) 177-206.

[3] C. Comi, A. Corigliano and G. Maier, Extremum properties of finite-step solutions in elastoplasticity with nonlinear mixed hardening, Internat. J. Solids Structures 27 (1991) 965-981.

[4] I. Hlaváček, J. Rosenberg, A.E. Beagles and J.R. Whiteman, Variational inequality formulation in strain space and finite element solution of an elasto-plastic problem with hardening, Comput. Methods Appl. Mech. Engrg. 94 (1992) 93-112. 
[5] J.F. Besseling, Models of metal plasticity: theory and experiment, in: A. Sawczuk and G. Bianchi, eds., Plasticity Today (Elsevier, London, 1984).

[6] M.J. Sewell, Maximum and Minimum Principles: A Unified Approach with Applications (Cambridge University Press, Cambridge, 1987).

[7] O. DeDonato, Fundamental of Elastic-Plastic Analysis, in: M.Z. Cohn and G. Maier, eds., Engineering Plasticity by Mathematical Programming (Pergamon Press, New York, 1979) 325-350.

[8] K. Schittkowski, NLPQL: A Fortran subroutine solving constrained nonlinear programming problems, Ann. Oper. Res. 5 (1985-1986) 485-500.

[9] J.E. Taylor and Janos Logo, Analysis and design of elastic/softening truss structures based on a mixed form extremum principle, in: G. Rozvany, ed., Optimization of Large Structural Systems (Kluwer, Dordrecht, 1993) 683-696.

[10] J.E. Taylor, Truss topology design for elastic/softening materials, in M.P. Bendsöe and C. Mota Soares, eds., Topology Design of Structures (Kluwer, Dordrecht, 1993) 451-468. 